\title{
Preparation and In Vitro Evaluation of a Multifunctional Iron Silicate@Liposome Nanohybrid for pH-Sensitive Doxorubicin Delivery and Photoacoustic Imaging
}

\author{
Zehua Liu, ${ }^{1}$ Shaoheng Tang, ${ }^{2}$ Zhiran Xu, ${ }^{1}$ Yingjun Wang, ${ }^{1}$ Xuan Zhu, ${ }^{1}$ Liang-cheng Li, \\ Wanjin Hong, ${ }^{1}$ and Xiumin Wang ${ }^{1}$ \\ ${ }^{1}$ School of Pharmaceutical Sciences, Xiamen University, Xiamen 361102, China \\ ${ }^{2}$ College of Chemistry and Chemical Engineering, Xiamen University, Xiamen 361005, China
}

Correspondence should be addressed to Xiumin Wang; wangxm@xmu.edu.cn

Received 30 November 2014; Revised 5 February 2015; Accepted 9 February 2015

Academic Editor: Zehra Durmus

Copyright (C) 2015 Zehua Liu et al. This is an open access article distributed under the Creative Commons Attribution License, which permits unrestricted use, distribution, and reproduction in any medium, provided the original work is properly cited.

For preventing premature drug release in neutral environment and avoiding them being trapped into the endosomal/lysosomal system, we developed a novel iron silicate@liposome hybrid (ILH) formulation, which can be used as a carrier to transport doxorubicin (DOX) in a pH-sensitive manner and to escape from endosomal/lysosomal trapping through "proton-sponge" effect. The high intensity of photoacoustic signal from in vitro photoacoustic imaging (PAI) experiments suggests that it is a promising candidate for PAI agent, providing the potential for simultaneously bioimaging and cancer-targeting drug delivery. Cytotoxicity of our formulation toward tumor cells was remarkably higher than free DOX $(48.4 \pm 7.7 \%$ and $26.2 \pm 8.4 \%, P<0.001)$. Confocal laser scanning microscopy experiments showed the enhanced transportation and enrichment process of DOX in QSG-7703 cells. Taking together, we developed an easy approach to construct a multifunctional anticancer drug delivery/imaging system with a potency as a PAI agent. The strategy of combining drug carrier and imaging agent is an emerging platform for further construction of nanoparticle and may play a significant role in cancer therapy and diagnosis.

\section{Introduction}

Doxorubicin (DOX), the most widely used chemotherapeutic drug, has deleterious side effects, including myelosuppression, gastrointestinal toxicity, and especially cardiotoxicity [1]. In order to minimize the side effects, using nanoparticles as carriers for DOX delivery has been explored in many years [2-5]. However, a great challenge still exists in the development of well-controlled DOX loaded nanoparticle. It should have the ability of enhancing DOX cellular uptake and releasing the drug within the cytoplasm triggered by environmental stimuli, so that DOX can reach the nucleus to achieve the desired therapeutic effects $[6,7]$. One obstacle is that DOX has been previously reported to undergo ion trapping in acidic conditions such as those present in the extracellular microenvironment of solid tumors ( $\mathrm{pH}$ 6.5-6.9) and especially in the internal milieu of endosomal/lysosomal
( $\mathrm{pH}$ 5.0-6.0) as the protonated DOX has a lower membrane permeability [8]. So a proper method should be applied to interrupt the endosomal/lysosomal system and allow the free DOX to escapes to the cytoplasm after nanoparticle being uptaken by the cell [9]. Meanwhile, this "zero-premature" could also diminish the side effects.

Besides of smart drug delivery, a desired nanoparticle system should also have the ability of bioimaging. Among all the imaging method, photoacoustic imaging (PAI), also known as optoacoustic imaging, is drawing increasing attention. It can generate high-definition volumetric images of tissue by measuring light-induced sound waves from its optically absorbing structures. Photoacoustic imaging is capable of real-time imaging and is especially suitable for continuous and repetitive imaging of disease sites for long-term monitoring of disease progression or therapeutic outcome [10-12]. To produce a preferred photoacoustic signal, several materials 
have been investigated for many years. Nanoparticle-based PAI uses gold nanoparticles, gold/silica hybrids, carbonbased particles, porphysomes, iron oxide nanoparticles, copper sulfide, and others. While nanoparticles do offer robust and stable photoacoustic signal, they are hampered by poor biodistribution and clearance profiles $[13,14]$. Generally, the most widely accepted method to enhance the circulation time is by modifying the nanoparticles with polyethylene glycol (PEG). PEG is a nontoxic, nonimmunogenic, protein resistant polymer and is widely used in pharmaceutical field. PEGylated nanoparticles improve stealth properties and prevent agglomeration of the particles, thereby providing protection against corrosion, evading reticuloendothelial system (RES). However, multistep modifying and fabricating processes are generally required to have several steps of chemical reactions which are complicated and time-consuming [15]. Besides, solvents and ligands applied in modifying processes may also be toxic to the cell.

Based on the problems above, we put our interests in silica based nanomaterials. Several advantages including high biocompatibility and easily modified surfaces make it a good candidate for various biochemical applications [1619]. Recent studies showed that DOX can form unstable complexes with metal ions such as $\mathrm{Fe}^{3+}$ and $\mathrm{Cu}^{2+}$, which will degrade in acidic environment [20-22]. So the concept of metal silicate nanostructure draws our attention. Herein, we prepared a novel DOX loaded nanohybrid by employing a novel $\mathrm{pH}$-sensitive nanoparticle with a PEGylated liposome. We prepared the iron silicate hollow nanoparticle through a modified hypothermal method. It has $\mathrm{Fe}^{3+}$ on its surface, by which DOX can only release in the acidic environment as the abundant $\mathrm{H}^{+}$in acidic environment will competitively bind to the nanoparticle. Our drug delivery system may also have the "proton-sponge" effect, as the binding between $\mathrm{H}^{+}$ and our nanoparticle may intervene the ion pump in endosomal/lysosomal system. As to the lipid selection, dioleoylphosphatidylethanolamine (DOPE) and soybean phosphatidylcholine (SPC) were used to prepare the liposome. DOPE was widely used in cationic liposome to meditate the gene transfer, as several studies suggested that it can largely enhance the transfection efficiency and is known as "helper lipid" $[23,24]$. Furthermore, it can also enhance the stability of the liposome by its amino group bonding with the inner core. This versatile method to anchor PEG to the system can also promote the circulation time and avoid the liver/spleen capture. Meanwhile, photoacoustic imaging in vitro suggests that it can produce a strong PA signal which makes it a potential candidate for further application as a PAI agent. All in all, this hybrid has a novel structure, a satisfied drug releasing, and transportation feature and shows a promising prospect of future application in PAI. We report here the preparation, properties, and in vitro activity of this formulation.

\section{Materials and Methods}

2.1. Materials. Iron(III) acetylacetonate Fe(acac) $)_{3}$, tetraethoxysilane (TEOS), and urea were purchased from Alfa Aesar. Soybean phosphatidylcholine (SPC) and dioleoylphosphatidylethanolamine (DOPE) were purchased from Shanghai
Advanced Vehicle Technology Co. Ltd. (Shanghai, China). $\mathrm{NH}_{2}-\mathrm{mPEG}_{2000}$ was obtained from Xiamen Sinopeg Biotech CO.LTD (Xiamen, China). N-hydroxysuccinimide (NHS), cholesteryl chloroformate, and dicyclohexylcarbodiimide (DCC) were purchased from J\&K Chemical Scientific Co. Ltd. (Beijing, China). Ethanol and concentrated ammonia solution $\left(28 \%-30 \% \mathrm{NH}_{3}\right.$ in water) were obtained from Sinopharm Chemical Reagent Co. Ltd. (Shanghai, China). Doxorubicin hydrochloride was purchased from HuaFeng United Technology Co. Ltd. (Beijing, China). QSG-7703 cells were purchased from cell storeroom of Chinese Academy of Sciences. All reagents were analytically pure and used as received without further purification.

\subsection{Preparation of the Drug Delivery System}

2.2.1. Synthesis and the Characterization of Chol-mPEG ${ }_{2000}$. Cholesteryl chloroformate was reacted with $\mathrm{NH}_{2}-\mathrm{mPEG}_{2000}$ (molar ratio $=2: 1)$ in dry dichloromethane $(\mathrm{DCM})$ at room temperature under argon in the presence of triethylamine (TEA) and 4-dimethylaminopyridine (DMAP) for about $8 \mathrm{~h}$. After thin layer chromatography (TLC) showed the disappearance of $\mathrm{NH}_{2}-\mathrm{mPEG}_{2000}$, the reaction mixture was filtered and the filtrate was evaporated under vacuum. The residue was purified on a silica-gel chromatography column (DCM : $\mathrm{MeOH}=8: 1$ ) to get Chol- $\mathrm{mPEG}_{2000}$ (Figure 1(a)).

${ }^{1} \mathrm{H}$ NMR, FTIR, and thermogravimetric analysis (TGA) were used to confirm the successful synthesis of Chol$\mathrm{mPEG}_{2000}$. FTIR spectroscopic studies of the $\mathrm{mPEG}_{2000^{-}}$ $\mathrm{NH}_{2}$, cholesteryl chloroformate, and Chol-mPEG 2000 were carried out in the operating range of $4000-600 \mathrm{~cm}^{-1}$, using a Jasco model 410 FTIR and KBr pellet technique. TGA analysis was carried out on TG 209 F1 (Netzsch Co. Ltd., Germany). The analysis was performed using platinum crucibles in nitrogen. The program was heating rate of $10^{\circ} \mathrm{C} / \mathrm{min}$ from 25 to $800^{\circ} \mathrm{C}$ and an isothermal stage of $5 \mathrm{~min}$ at $800^{\circ} \mathrm{C}$. A flow rate of $100 \mathrm{~cm}^{3} / \mathrm{min}$ of nitrogen was maintained during the analyses.

2.2.2. Preparation of the Inner Iron Silicate Core of the System. Hollow iron silicate nanospheres were prepared using silica nanoparticle as the template and Iron(III) acetylacetonate as iron ligand according to the previously described procedure with a slightly change of reaction condition [25]. The $\mathrm{SiO}_{2}$ templates were prepared in a modified Stöber method. We use the following method to synthesis $\mathrm{SiO}_{2}$ template with the size of $75 \mathrm{~nm}$. Firstly, $3.15 \mathrm{~mL}$ concentrated ammonia and $1 \mathrm{~mL}$ water were added to $60 \mathrm{~mL}$ ethanol in sequence. After stirring for $30 \mathrm{~min}, 2.3 \mathrm{~mL}$ tetraethoxysilane was added. The reaction mixture was further stirred for $6 \mathrm{~h}$ under room temperature. The resultant colloids were centrifuged (18,000 rcf), fully washed by ethanol and water (50:50 volume ratio), then redispersed in distilled water. Then $25 \mathrm{mg}$ sphere dispersed in $10 \mathrm{~mL}$ water containing $1.0135 \mathrm{~g}$ urea was mixed with $88 \mathrm{mg} \mathrm{Fe}(\mathrm{acac})_{3}$ in $5 \mathrm{~mL}$ ethanol following with ultrasonic treatment. The suspension was transferred into a Teflon-lined stainless autoclave with a capacity of $25 \mathrm{~mL}$, kept at $190^{\circ} \mathrm{C}$ for $48 \mathrm{~h}$, and then cooled to ambient temperature to get the hollow iron silicate spheres (HISS). 
<smiles>CC(C)CCCC(C)[C@H]1CC[C@H]2[C@H]3CC=C4C[C@H](OC(=O)Cl)CC[C@]4(C)[C@H]3CC[C@@]21C</smiles>

Cholesteryl chloroformate

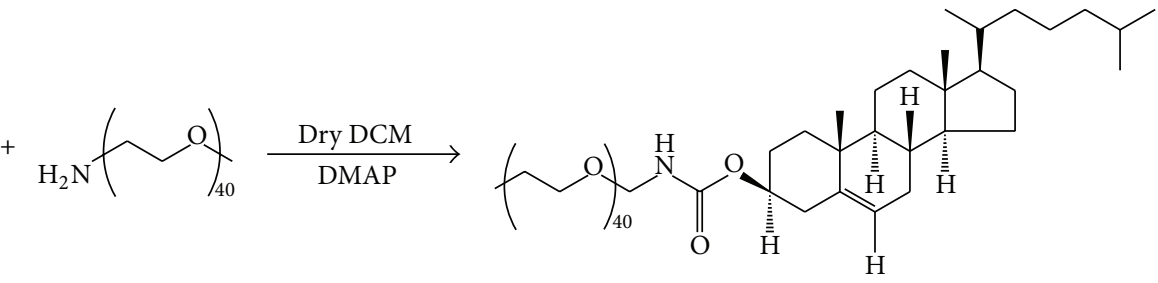

$\mathrm{mPEG}_{2000}-\mathrm{NH}_{2}$

Chol-mPEG 2000 (a)

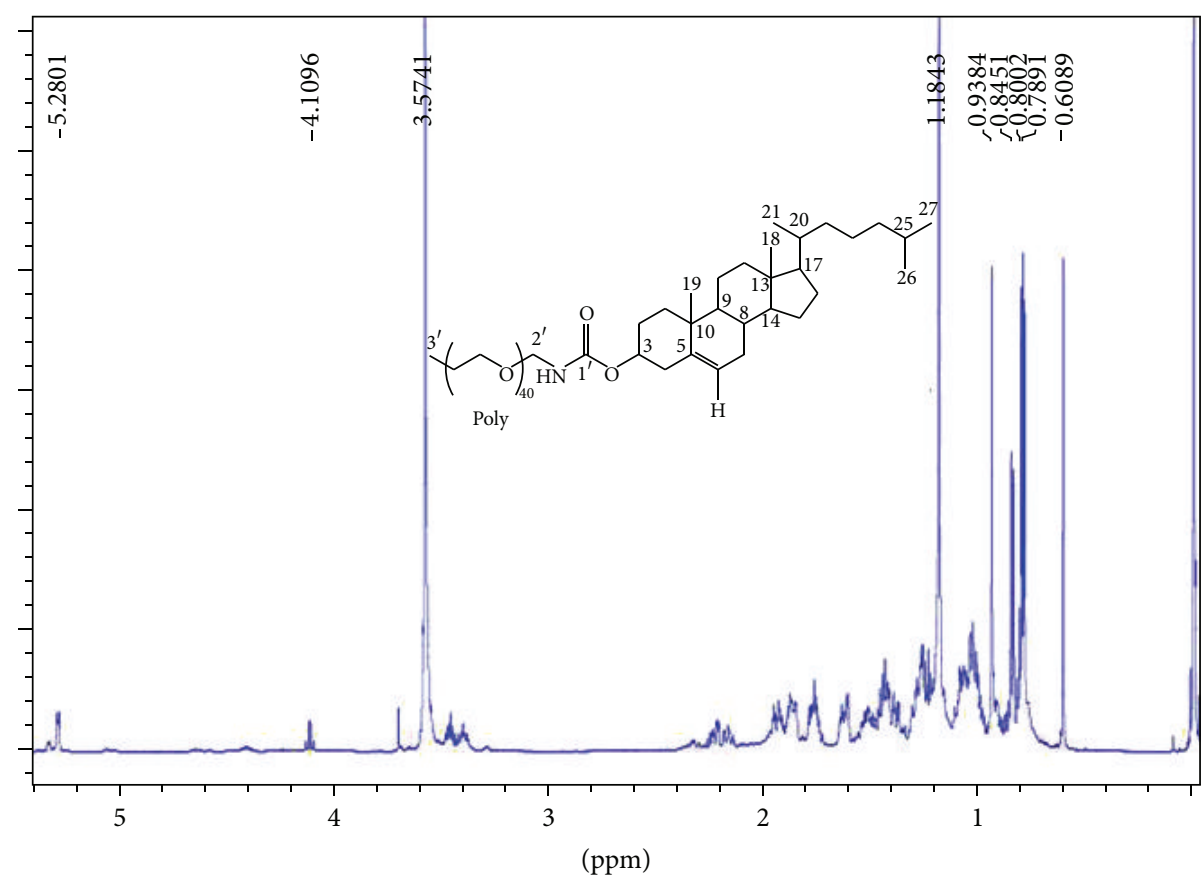

(b)

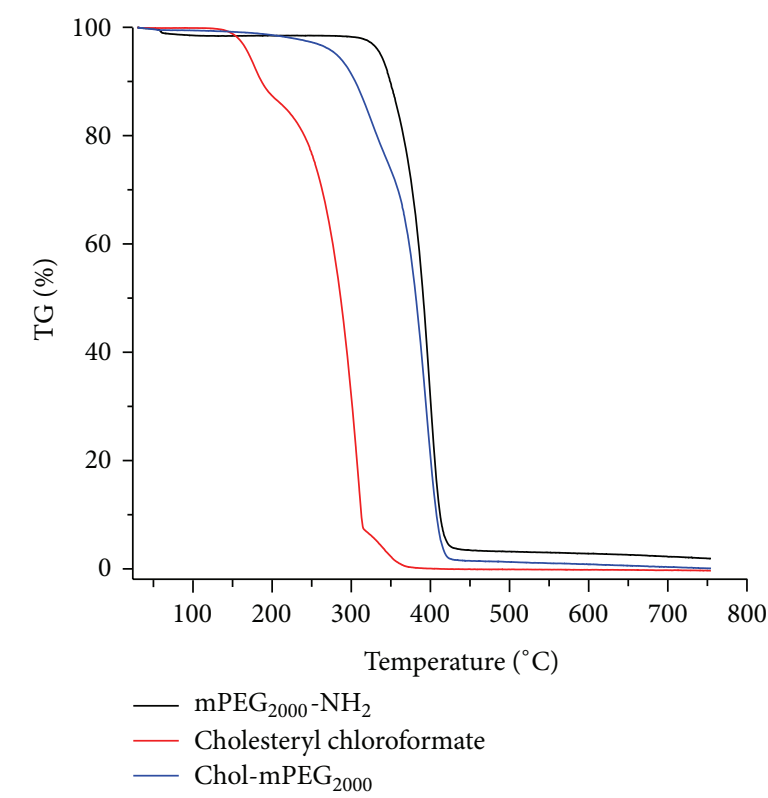

(d)

FIGURE 1: (a) Schematic of synthesis of Chol-mPEG ${ }_{2000}$; (b) ${ }^{1} \mathrm{H}$ NMR spectra of Chol-mPEG ${ }_{2000}$; (c) FTIR spectra of $\mathrm{mPEG}_{2000}-\mathrm{NH}_{2}$, cholesteryl chloroformate, and Chol-mPEG 2000 ; (d) TGA curve of $\mathrm{mPEG}_{2000}-\mathrm{NH}_{2}$, cholesteryl chloroformate, and Chol-mPEG $\mathrm{m}_{2000}$. 
Mix $2 \mathrm{mg}$ HISS and $1 \mathrm{mg}$ DOX in $2 \mathrm{~mL}$ water to stir for $12 \mathrm{~h}$. The products were collected using centrifugation at $18,000 \mathrm{rcf}$. The supernatant was collected to measure the unloaded DOX. Then the final particles were dissolved in $1 \mathrm{~mL}$ water for further modification.

2.2.3. Preparation of Liposome and the Final Iron Silicate@Liposome Nanohybrid (ILH). Thin film dispersion method was used to prepare the liposome. Generally, $11.7 \mathrm{mg} \mathrm{SPC}$, $8.7 \mathrm{mg}$ DOPE, $2.8 \mathrm{mg}$ cholesterol, and $5.4 \mathrm{mg}$ Chol-mPEG $\mathrm{m}_{2000}$ (molar ratio $1.5: 1.5: 1: 0.4$ ) were dissolved in a mixed solvent of chloroform and methanol (volume ratio $4: 1$ ). The solvent was removed completely in a rotary evaporator under vacuum above the transition temperature of the lipid. The lipid film was dried further for $1 \mathrm{~h}$ under a vacuum. After vacuum drying and desiccation, PBS solution ( $\mathrm{pH}$ 7.4) was added for hydration. The suspension was sonicated (input power of $20 \%$ for 210 seconds in total) to reduce the particle size and the resulting liposomes were extruded through a $0.22 \mu \mathrm{m}$ filter membrane to get the blank liposome.

The obtained $2 \mathrm{mg}$ HISS core dispersed in water was mixed with liposome ( $2 \mathrm{mmol}$ total lipid) to incubate overnight to get the final ILH.

2.3. Characterization. Transmission electron microscopy (TEM) studies were performed on a TECNAI F-30 high resolution transmission electron microscope operating at $300 \mathrm{kV}$. TEM samples were prepared by dropping $5 \mu \mathrm{L}$ of HISS particle dispersion in distilled water on amorphous carbon coated copper grids and drying under vacuum overnight. For ILH, 1\% phosphotungstic acid was used to stain the ILH for $1 \mathrm{~min}$ before the observation with TEM. The zeta potential and particle size of HISS, blank liposome, and final ILH were determined in $1 \mathrm{mM} \mathrm{KCl}$ by a Malvern Zeta Sizer Nano series (Westborough, MA).

2.4. Drug Release Study. The drug release profile was evaluated at different $\mathrm{pH}$ value. The PBS buffer was adjusted to different $\mathrm{pH}$ value by $\mathrm{HCl}$, and the drug loaded delivery system was dissolved in PBS at $\mathrm{pH} 7.4$ for $6 \mathrm{~h}$. The solution was centrifuged $(18,000 \mathrm{rcf})$ at each hour, the supernatant was obtained, and EnVision Multilabel Reader (EnVision, USA) was used to measure the intensity of fluorescence to get the amount of DOX at each time point (excitation wavelength: $490 \mathrm{~nm}$, emission wavelength: $550 \mathrm{~nm})$. Then the solution was changed into acidic PBS $(\mathrm{pH}=5)$, and the same procedure was conducted to measure the releasing curve.

Furthermore, same amount of DOX loaded ILH was dispersed in PBS solution at the $\mathrm{pH}$ value of $7.4,6$, and 5 to detect the relationship between releasing amount of DOX and the $\mathrm{pH}$ value.

2.5. Acid-Base Titration. The buffering capacity of the ILH was determined by an acid-base titration assay over the $\mathrm{pH}$ range from 12.0 to 2 . First, ILH solution was diluted to a final concentration of $0.5 \mathrm{mg} / \mathrm{mL} 5 \mathrm{~mL}$ of distilled water solution. The $\mathrm{pH}$ values of the sample solutions were adjusted to 12.0 by $1 \mathrm{M} \mathrm{NaOH}$, before $0.1 \mathrm{M} \mathrm{HCl}$ was successively added to the above solutions until the $\mathrm{pH}$ value reached 2 , the changes in $\mathrm{pH}$ being monitored by a $\mathrm{pH}$ meter (Model 320, Delta).

2.6. Photoacoustic Imaging In Vitro. Photoacoustic imaging (PAI) was performed using Nexus 128 scanner manufactured by Endra Life Sciences (Ann Arbor, MI, USA). $808 \mathrm{~nm}$ was chose as the working laser wavelength with 30 pulses averaging. During the scanning process, the water heating system maintains the water temperature at $38^{\circ} \mathrm{C}$. Different concentrations of ILH were prepared and placed in a plastic thin film suspending in the water-filled plastic tube. The photoacoustic signal was measured and collected for analysis.

\subsection{In Vitro Study}

2.7.1. Cell Culture. The human hepatoma cells QSG-7703 were cultured in RPMI-1640 containing 10\% (v:v) fetal bovine serum (FBS). $100 \mathrm{U} / \mathrm{mL}$ penicillin and $100 \mathrm{mg} / \mathrm{mL}$ streptomycin were also added. Cells were cultured in an incubator (Thermo Scientific, USA) at $37^{\circ} \mathrm{C}$ under an atmosphere of $5 \% \mathrm{CO}_{2}$ and $90 \%$ relative humidity and were subcultivated approximately every 3 days at $80 \%$ confluence using $0.25 \%$ $(\mathrm{w}: \mathrm{v})$ trypsin at a split ratio of $1: 3$.

2.7.2. In Vitro Cytotoxicity Assay. To study the cytotoxicity of ILH, QSG-7703 cells were seeded in a 96-well plate at a density $10^{4}$ cells/well for $24 \mathrm{~h}$ at $37^{\circ} \mathrm{C}$ in $5 \% \mathrm{CO}_{2}$. After $24 \mathrm{~h}$ of culture with the free DOX, blank ILH, and DOX-loaded ILH at several concentrations $(2 \mu \mathrm{g} / \mathrm{mL}, 5 \mu \mathrm{g} / \mathrm{mL}, 10 \mu \mathrm{g} / \mathrm{mL})$, the medium was removed and $75 \mu \mathrm{L}$ of $\operatorname{MTT}(3-(4,5)-$ dimethylthiazol-2-yl)-2,5-diphenyltetrazolium bromide) dye solution $(5 \mathrm{mg} / \mathrm{mL}$ PBS solution $\mathrm{pH}=7.4$ ) was added to each well and incubated for $4 \mathrm{~h}$ at $37^{\circ} \mathrm{C}$ and $5 \% \mathrm{CO}_{2}$. The medium was removed and formazan crystals were solubilized with $150 \mu \mathrm{L}$ of dimethyl sulfoxide (DMSO). Absorbance of each well was read using a spectrophotometer (Biotek, USA) at $490 \mathrm{~nm}$ and the relative cell viability (\%) related to control wells containing cell culture medium without nanoparticles was calculated by (Abs sample/Abs control) $\times 100 \%$. The results are presented as mean \pm standard deviation.

2.7.3. Confocal Fluorescence Imaging. $1 \times 10^{4}$ QSG-7703 cells per well were cultured in a 12 -well chamber slide and incubated with DOX, DOX-loaded ILH. After $4 \mathrm{~h}$ and $8 \mathrm{~h}$, the cells were washed with phosphate buffered saline (PBS) $\times 3$, fixed with $4 \%$ paraformaldehyde for $30 \mathrm{~min}$ and stained with $4^{\prime}$,6-diamidino-2-phenylindole (DAPI, $1 \mu \mathrm{g} / \mathrm{mL}$ in PBS) for $5 \mathrm{~min}$. After another washing with PBS $(\times 3)$, dishes were covered with glycerol following with fixing on the slide. The fluorescence images were acquired by confocal laser scanning microscopy (CLSM) (Leica TCS SP5) with the excitation wavelength of $488 \mathrm{~nm}$ and emission wavelength between 530 and $600 \mathrm{~nm}$.

2.8. Statistical Analysis. The drug loading and release tests were repeated 3 times and the results are given as mean \pm standard deviation (SD). The cytotoxicity experiments were repeated 6 times and the results are presented as mean \pm SD. Statistical significance was calculated using a two tailed Student's $t$-test and $P<0.05$ was considered significant. 


\section{Results and Discussion}

3.1. Synthesis and Characterization of Chol-mPEG ${ }_{2000} \cdot{ }^{1} \mathrm{H}-$ NMR was used to confirm the successful synthesis of Chol$\mathrm{mPEG}_{2000}:{ }^{1} \mathrm{H}-\mathrm{NMR}\left(\mathrm{CDCl}_{3}, 400 \mathrm{MHz}\right): \delta 0.61(3 \mathrm{H}, \mathrm{s}, \mathrm{H}-18)$, $0.79(3 \mathrm{H}, \mathrm{d}, \mathrm{H}-26, J=2.7 \mathrm{~Hz}), 0.80(3 \mathrm{H}, \mathrm{d}, \mathrm{H}-27, J=2.7 \mathrm{~Hz})$, $0.85(3 \mathrm{H}, \mathrm{d}, \mathrm{H}-21, J=6.6 \mathrm{~Hz}), 0.94(3 \mathrm{H}, \mathrm{s}, \mathrm{H}-19), 1.18(3 \mathrm{H}, \mathrm{s}$, H-3'), 3.48-3.81 (m, H-poly), 3.57 (3H, overlaps, H-2 ${ }^{\prime}$ ), 4.11 (q, $1 \mathrm{H}, \mathrm{H}-3, J=7.1 \mathrm{~Hz}$ ), 5.28 (t, 1H, H-6, $J=2.6 \mathrm{~Hz}$ ) (Figure 1(b)). Purification of the final product was detected by thinlayer chromatographic silica gel, and no other spots can be detected at series of developers. Final yield of this reaction was $77.93 \%$.

As it is shown in Figure 1(c), for PEG, peak at $2890 \mathrm{~cm}^{-1}$ was attributed to the stretching vibration of $-\mathrm{CH}_{2}-$; peaks around $1108 \mathrm{~cm}^{-1}$ were assigned to helix and planar-zigzag conformation of $\mathrm{OCH}_{2}-\mathrm{CH}_{2} \mathrm{O}$ structure. The spectrum of Chol-mPEG 2000 was similar with PEG; in addition, new bands appeared at $1732 \mathrm{~cm}^{-1}$, which is in the characteristic region for $\mathrm{C}=\mathrm{O}$ vibrations $[26,27]$. This confirmed the successful introduction of carbamate groups due to the transformation of the original chloroformate in cholesteryl chloroformate.

As it is shown in Figure 1(d), the process of degradation of the pure $\mathrm{mPEG}_{2000}-\mathrm{NH}_{2}$ has two steps. The first step, minor weight loss step, is the elimination of weakly bound water molecules at temperature up to $100^{\circ} \mathrm{C}$. The second weight loss comes sharply from 350 to $425^{\circ} \mathrm{C}$ due to the $\mathrm{mPEG}_{2000^{-}}$ $\mathrm{NH}_{2}$ main chain degradation occurred [28]. As to cholesteryl chloroformate, most of its degradation occurred below $330^{\circ} \mathrm{C}$. However, when the temperature heated up to $330^{\circ} \mathrm{C}$, Chol$\mathrm{mPEG}_{2000}$ lost about $19.7 \%$ of its total weights. Considering the molecular weight of cholesteryl chloroformate (449) and $\mathrm{mPEG}_{2000}-\mathrm{NH}_{2}$ (around 2000), we can conclude that the first stage of weight loss of Chol-mPEG 2000 is mainly due to the degradation of cholesteryl group in Chol-mPEG $\mathrm{P}_{2000}$. After the temperature was heated above $330^{\circ} \mathrm{C}$, the degradation curve of Chol-mPEG 2000 was similar to pure PEG. So the results of TGA can also confirm the successful linkage between $\mathrm{mPEG}_{2000}-\mathrm{NH}_{2}$ and cholesteryl chloroformate.

3.2. Characterization. The original nanoparticles were prepared in a facile hydrothermal reaction through a sacrificialtemplating process. As shown in Figure 2(a), the obtained nanoparticles had an average diameter of $100 \mathrm{~nm}$. The robust and rough surface were formed by small particles, which increased the total surface areas and interfacial $\mathrm{Fe}^{3+}$ amount to bond with $\mathrm{NH}_{3}{ }^{+}$strongly. This coordination bonds can accomplish the drug loading, and the cavity of the nanoparticle shows a promising potentiality for multiple usage.

After modification, the final ILH was prepared. The DLS results suggested that the overall size of ILH was $141 \mathrm{~nm}$ in diameter with a narrow hydrodynamic diameter distribution (Figure 2(c)). Besides, the increased size (from $120 \mathrm{~nm}$ to $141 \mathrm{~nm})$ can also demonstrate the successful construction of ILH. Generally, particles near $100 \mathrm{~nm}$ in diameter tend to represent an optimal range for leveraging the EPR effect and minimizing clearance which gives our system a better potential for further usage [29]. Zeta potential $(\xi)$ was also measured to determine the structure (Figure 2(d)). The naked HISS has a zeta potential of $-30.2 \mathrm{mV}$, but when it was coated with liposome the zeta potential promoted to $-12.4 \mathrm{mV}$ which illustrating the successful construction of ILH. By coating the PEGylated liposome not only fluidity was enhanced, but also it has further meaning in the biocompatibility and longcirculation prospect. As zeta potential is an influential physical factor impacting pharmacokinetics and biodistribution, it is already known that negative particles $(\xi<-10 \mathrm{mV})$ exhibit strong RES uptake and adsorb proteins with a $\mathrm{pI}>5.5$, such as $\operatorname{IgG}$, and positive particles $(\xi>+10 \mathrm{mV})$ will induce serum protein aggregation which will further stimulate the clearance by the Kupffer cells from the blood circulation, and neutral nanoparticles (within $\pm 10 \mathrm{mV}$ ) exhibit the least RES interaction and the longest circulation. The former result suggested that ILH with liposome had a neutral zeta potential, which makes it a better candidate for the delivery system $[30,31]$. And comparing to the chemical modification, the introduction of PEG simply by insertion in the bilayer of the liposome can be less time-consuming and biosafety can be also enhanced.

The DOX loading ability of ILH was then studied. The loading content was evaluated by monitoring the fluorescence of the DOX solutions before and after treated with ILH. And result showed that the loading efficacy of the final ILH was about $21 \%(\mathrm{w}: \mathrm{w})$.

3.3. pH-Sensitive Drug Release Study. The suitable size originated EPR effect of ILH will facilitate its accumulation around tumor site. However, if the drug was released in this stage, it still has to face the obstacle of ion trapping. That is, the free DOX with a lower membrane permeability will have a limited ability of penetrating into the cell. So it is more desired that the nanoparticle was uptaken by the cell and entrapped in the endosomal/lysosomal system, and most of the drug will be released in this more acidic environment $(\mathrm{pH}$ from 5 to 6 ). To determine whether our ILH have controlled release feature specifically in endosomal/lysosomal system, the following experiments were performed. As it is shown in Figure $3(\mathrm{a})$, at the $\mathrm{pH}$ value of 7.4 , only $10.2 \pm 3.9 \%$ of the total loaded DOX was released within $3 \mathrm{~h}$, and nearly no DOX was further released in the following 3 hours. Totally, there was only $15.5 \pm 3.6 \%$ of the loaded drug was leaked in 6 hours. Then after dropping the $\mathrm{pH}$ value of solution into 5 , as we expected, there was a burst release within 4 hours, and nearly $80.5 \pm 7.3 \%$ of the drug was released from the system, indicating that the release kinetic is related to acidic $\mathrm{pH}$ value. As the firstly released DOX can be explained by surface absorbed free drug by Van der Waals' forces, other DOX molecules loaded are bound to HISS through $-\mathrm{NH}_{2}$ $\mathrm{Fe}^{3+}$ coordination bonds which are fairly stable under neutral condition. Therefore DOX coordinated on HISS can hardly be released unless under attacks by acids. And the results suggest that there is still a big amount of DOX anchored to the particle by coordination bond and avoid untargeted release. This feature also gives ILH potentiality of long-period store without further release. It also should be noted that a sustained release of DOX from the ILH may be important in achieving long-term therapeutic results and thus could 


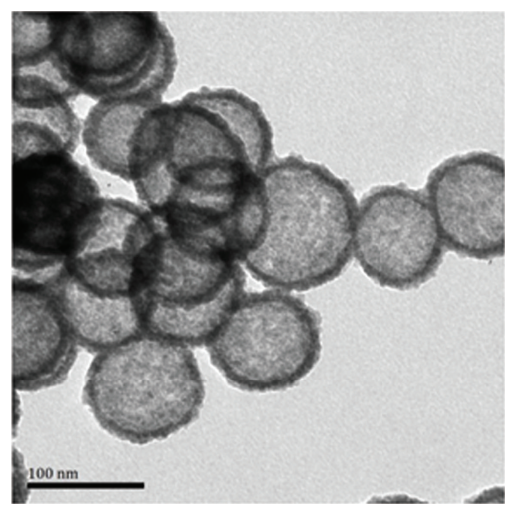

(a)

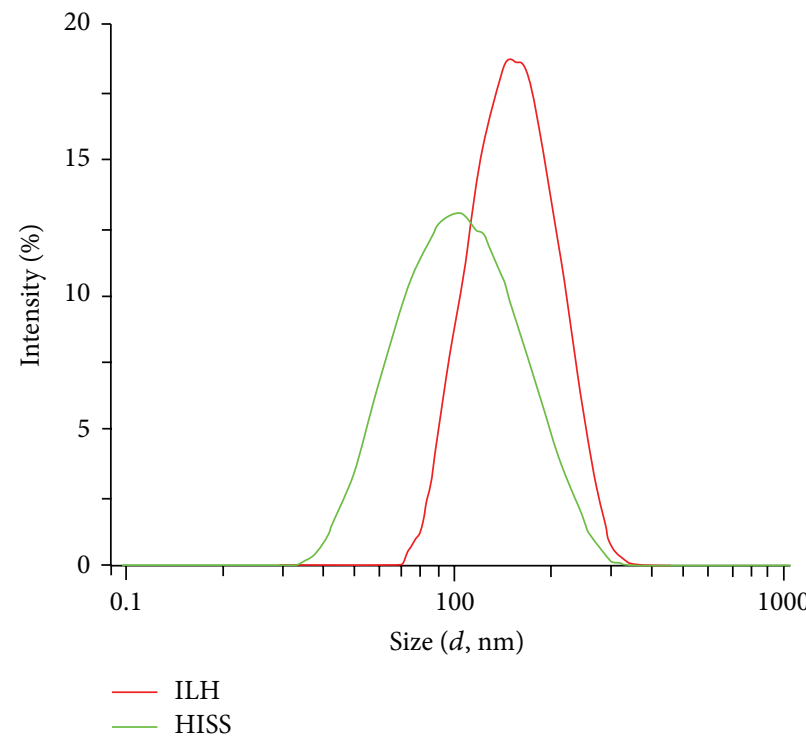

(c)

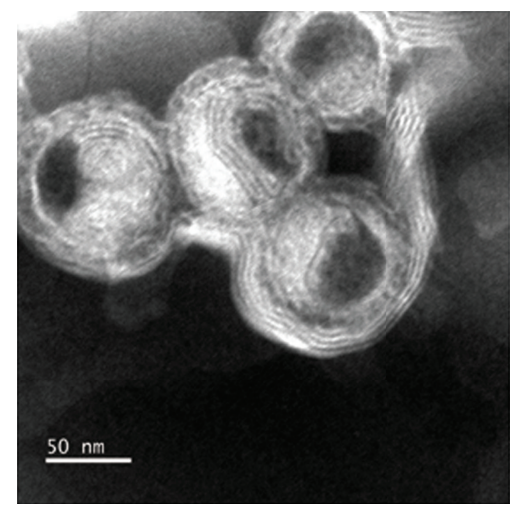

(b)

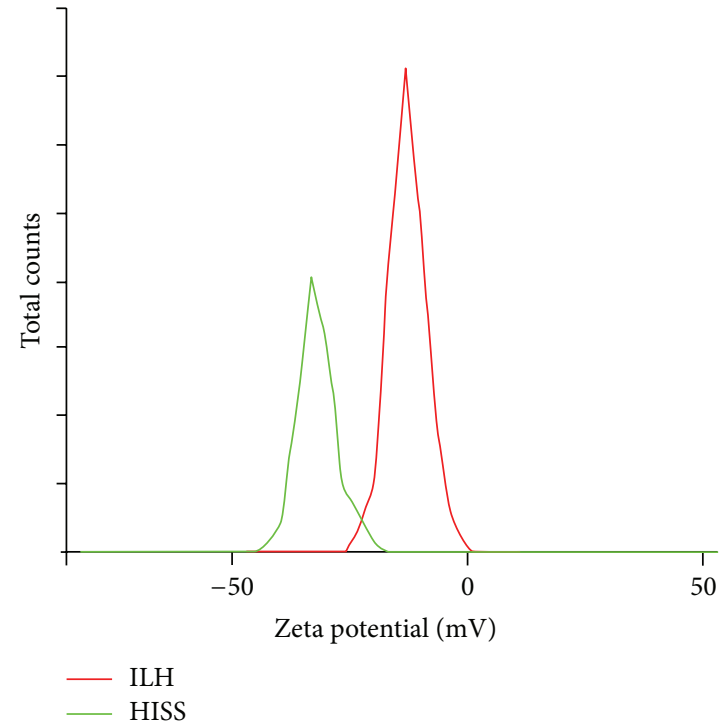

(d)

FIGURE 2: Characterization of our drug delivery system, representative (a) TEM image of HISS and (b) ILH with negative staining. (c) the change of hydrodynamic diameter distribution of bare HISS and ILH in water dispersion; (d) the change of zeta potential of bare HISS and ILH in water dispersion.

be beneficial in reducing side effects by decreasing the dose and/or the number of drug administrations.

We assume that the mechanism for this $\mathrm{pH}$-dependent release manner is that the $\mathrm{H}^{+}$can competitively bond with the nanoparticle. In that case, the concentration of $\mathrm{H}^{+}$should have a positive effect on releasing efficacy. Therefore we should observe the relationship between $\mathrm{pH}$ value and the drug release kinetics, and this " $\mathrm{H}^{+}$competitive bonding" $\mathrm{pH}$ sensitive mechanism will be briefly confirmed. The release curve under three different $\mathrm{pH}$ conditions were evaluated, and as it is shown in Figure 3(b), we found out that the release kinetics presented an acid-dependent tendency; that is, with the decrease of $\mathrm{pH}$ value, there was an obviously increase in the released DOX amount. When the $\mathrm{pH}$ value is 7.4, only about $15.5 \pm 4.5 \%$ of the loaded drug was released within 24 hours; however the releasing amount was dramatically increased when the $\mathrm{pH}$ value changed into acidic condition, and the release rate of DOX-loaded ILH under the $\mathrm{pH}$ condition of 5 and 6 was $80.1 \pm 6.2 \%$ and $47.3 \pm 4.4 \%$, respectively. Besides preliminary proving the mechanism, it is also reasonable to conclude that comparing to less acidic extracellular microenvironment of tumor, more free DOX will be released in the endosomal/lysosomal system after ILH was uptaken by the cells, so the ion trapping of the cell membrane can be avoided.

3.4. Proton Sponge Effect of ILH. Even though ILH has a desired releasing feature in extracellular microenvironment of tumor, when free drug was released in endosomal/lysosomal system, it also has to face the ion trapping of the endosome membrane. Otherwise it will end up with being degraded in the system and lost its chance to finally locate in the nucleus. So whether ILH has the ability to achieve the endosomal/lysosomal escape is also crucial for this delivery system. Generally, proton sponge effect was applied to accomplish this goal. Proton sponge effect is a phenomenon that 

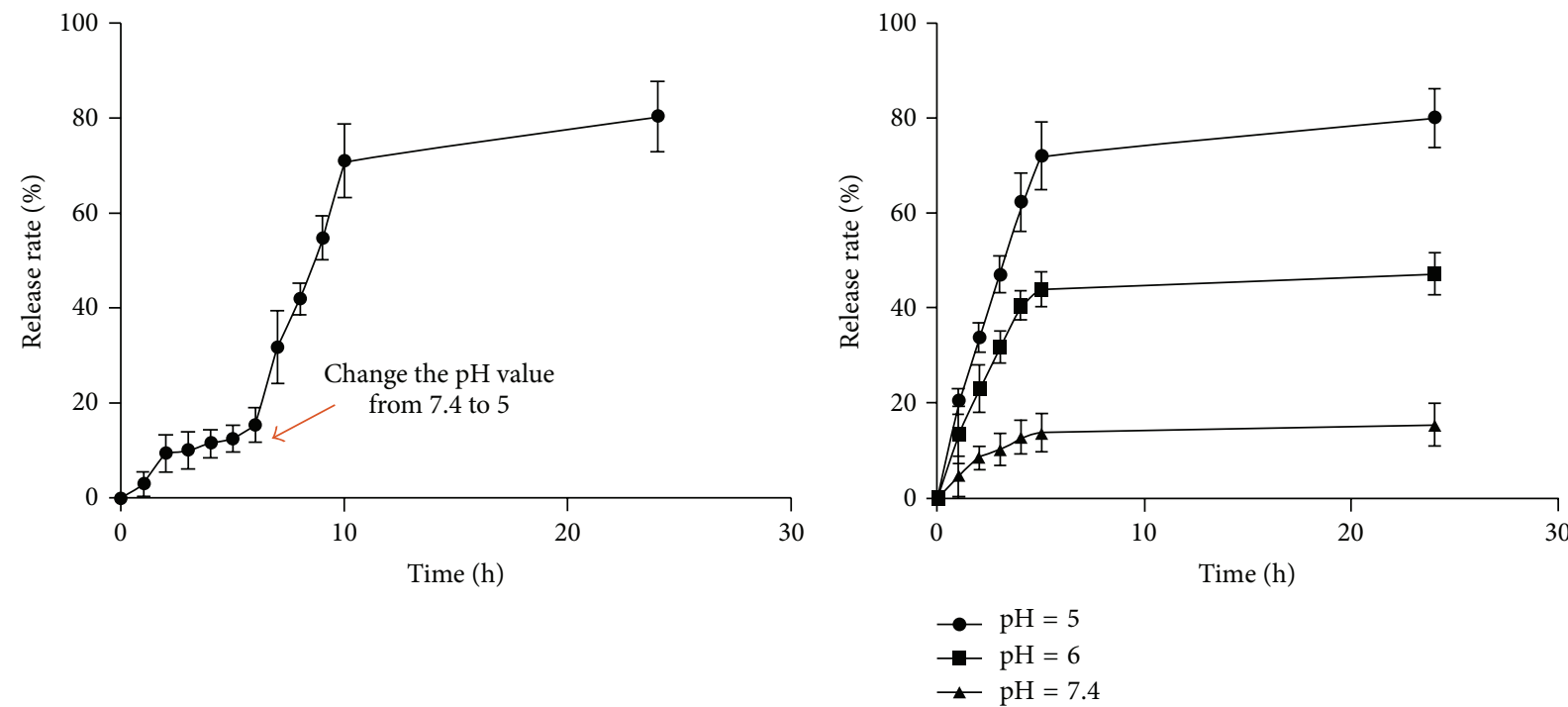

(a)

(b)

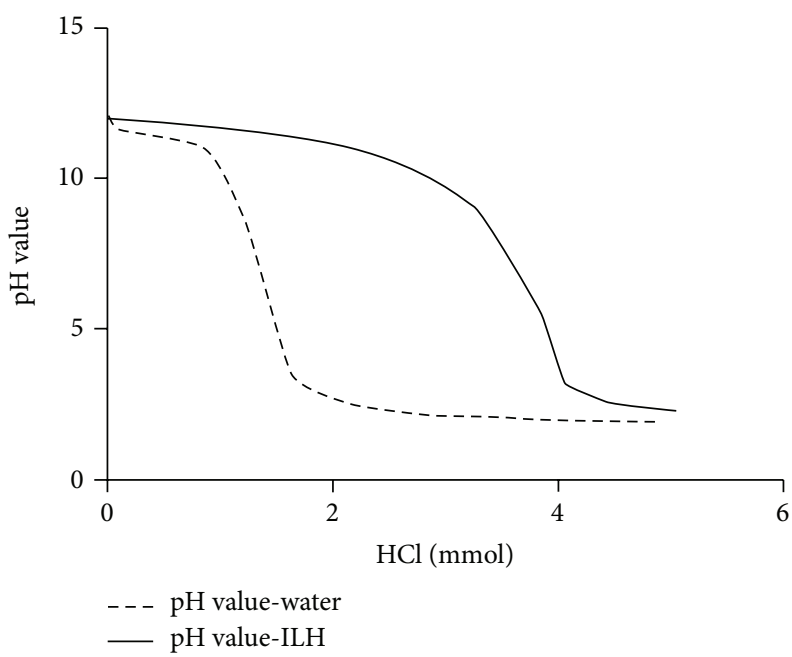

(c)

FIgURE 3: Acidic-dependent drug releasing feature: (a) $\mathrm{pH}$-sensitive release kinetics of DOX from ILH in PBS buffer at two different $\mathrm{pH}$ values $(n=3)$. (b) Sustained release kinetics of DOX from ILH in PBS buffer at different pH values $(n=3)$. (c) Buffering capacity of ILH indicating that our system has proton sponge effect.

when the drug delivery system enters the cell through endocytosis and stays in the endosome, it can absorb abundant $\mathrm{H}^{+}$, and the amount of $\mathrm{H}^{+}$in endosome will sharply decreased by this process. In order to maintain the electric neutrality of the endosomal/lysosomal system, proton pump will keep functioning and leads to the abundant retention of $\mathrm{Cl}^{-}$ion and water molecule per proton $[32,33]$. Subsequently the endosome swelling and rupture lead to the contents releasing into cytoplasm. From the results and former experiments, we assume that this $\mathrm{pH}$-sensitive feature is accomplished by the mechanism that protons would compete with loaded drug molecules to bind with metal ions, and the $\mathrm{pH}$-dependent profile could really imitate the proton sponge effects because the speed of proton binding process is enhanced with the big drop in $\mathrm{pH}$ value when the ILH is entrapped by endosome [34]. To further confirm the releasing mechanism of ILH and that ILH has the "proton sponge" effect, the buffering capacity of ILH was studied by acid-base titration. From the titration curves shown in Figure 3(c), we can conclude that a significant higher volume of acid is necessary to achieve the same $\mathrm{pH}$ value when the ILH was present, revealing the buffering action of ILH. This feature may lead to an additional pumping of protons into the endosomal/lysosomal vesicles followed by an influx of chloride anions (to maintain electric neutrality), so an increase in osmotic pressure will be achieved. This will finally cause the disbalance of the endosomal/lysosomal compartments and accelerate their rupture to release DOX. All in all, in addition to a better targeting 


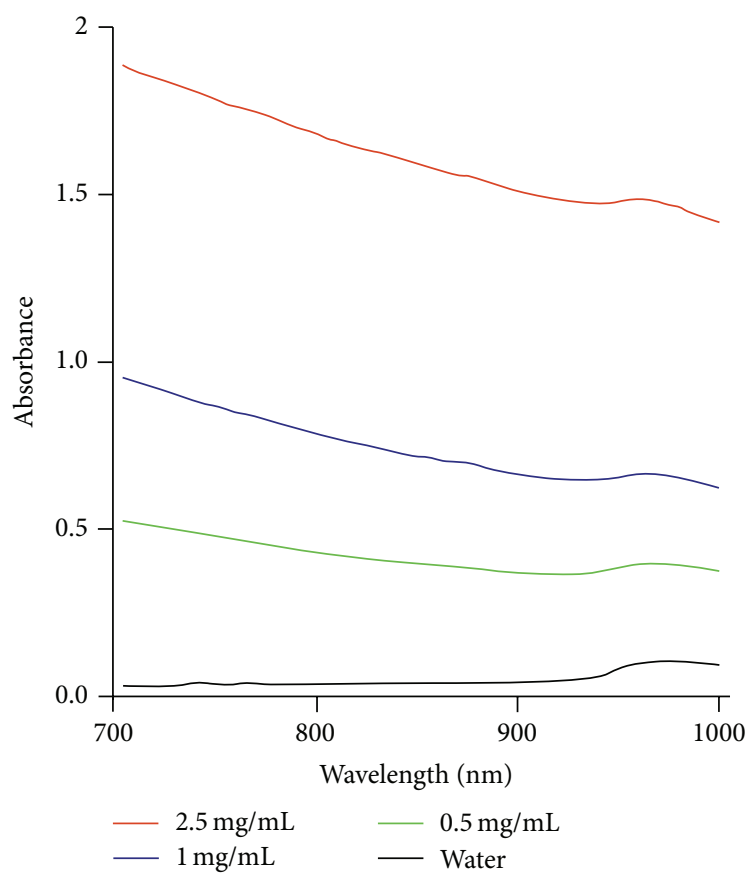

(a)

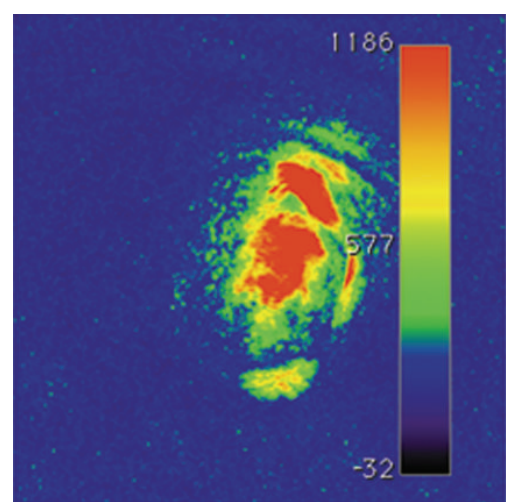

$5 \mathrm{mg} / \mathrm{mL}$

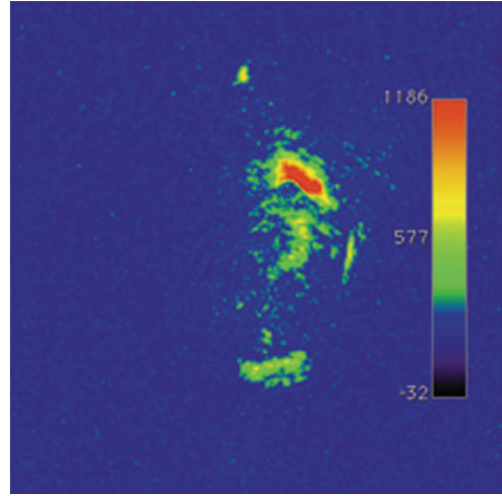

$0.5 \mathrm{mg} / \mathrm{mL}$

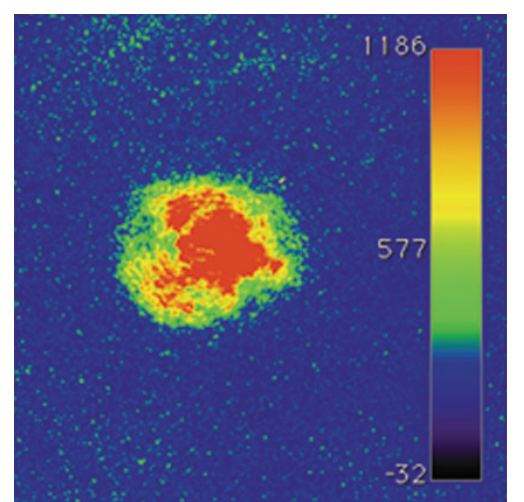

$2.5 \mathrm{mg} / \mathrm{mL}$

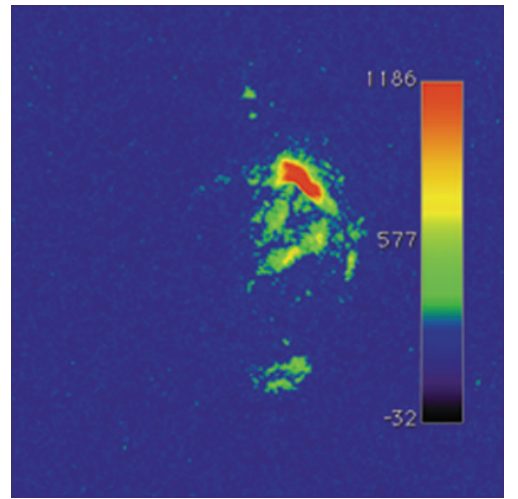

$0.25 \mathrm{mg} / \mathrm{mL}$

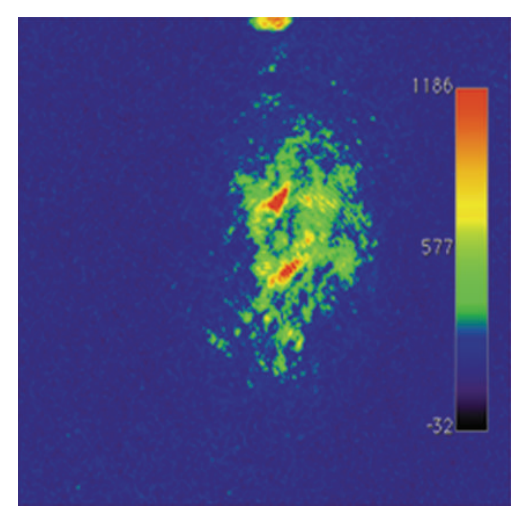

$1 \mathrm{mg} / \mathrm{mL}$

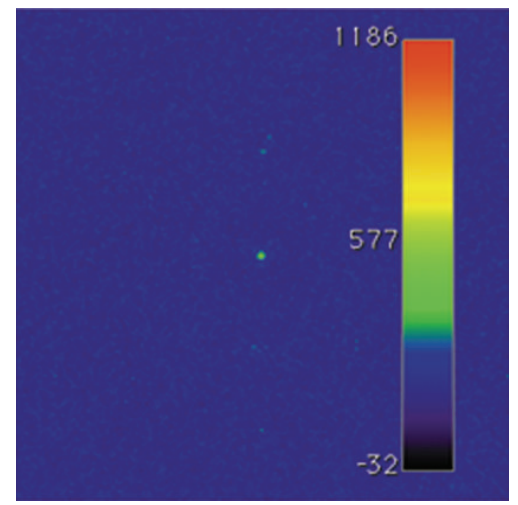

Water

(b)

FIGURE 4: Photoacoustic features of ILH (a) NIR absorption feature of ILH at different concentration. (b) PAI signal in vitro suggests its strong contrasting ability. 


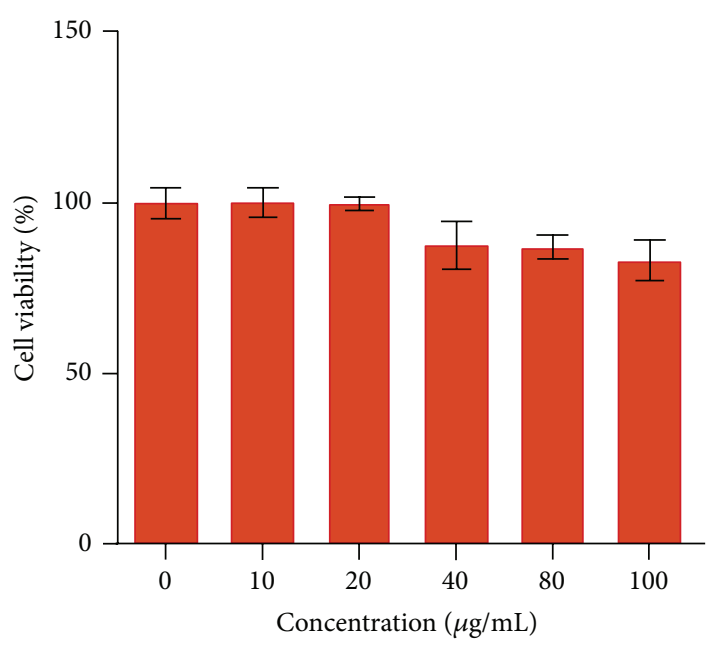

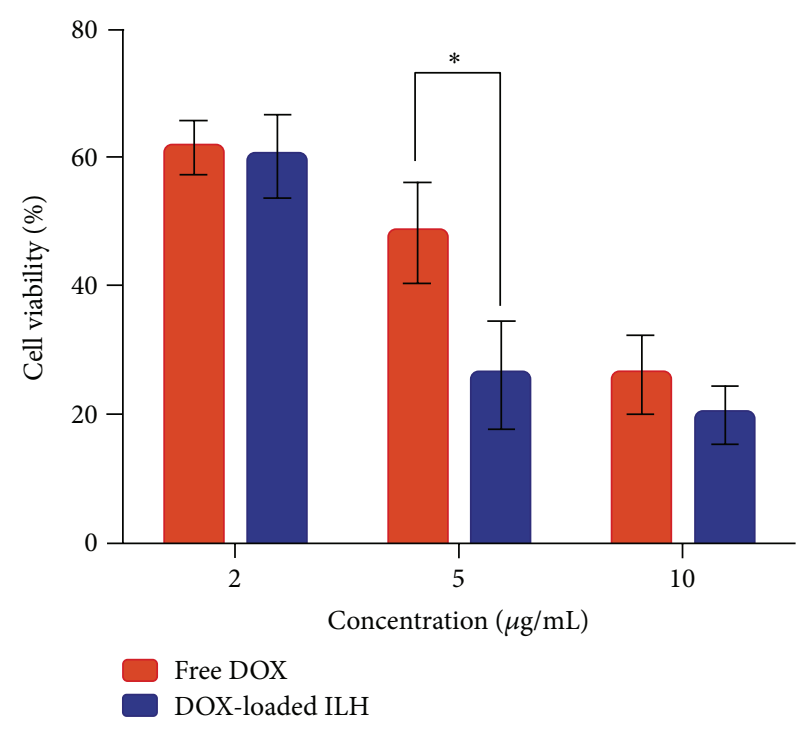

(b)

FIGURE 5: (a) Relative cell viability data of QSG-7703 cells incubated with DOX-free ILH at different concentrations, indicating the good biocompatibility of this nanohybrid. (b) Relative cell viability data of QSG-7703 cells incubated with free DOX and DOX-loaded ILH at different concentrations ( ${ }^{*}$ significant at $0.1 \%$ level).

release profile, this character of $\mathrm{pH}$-dependent release may contribute to endosomal/lysosomal escape which gives ILH a better cellular-uptake efficacy.

3.5. Photoacoustic Imaging of ILH In Vitro. To investigate this material as a PAI agent, we first need to know whether our ILH is in favor of using light as an excitation source and optimize the imaging wavelength. It is well known that generally the particle should have strong absorption of near infrared (NIR) light to produce PA signal, and it always presents as morphology-dependent and concentration-dependent manner [35]. The primary universal mechanism for PAI is photothermal. After the introduction of laser, the light energy is absorbed by the nanoparticles and converted to heat. The heat causes the nanoparticle to undergo rapid thermo elastic expansion and then generates acoustic signal that originates from the photoabsorber following by being detected with a conventional ultrasound (US) transducer [36]. As the precondition for PAI is the absorbance for lights, so we conduct a full wave scanning to find out whether our particle has NIR absorption. As can be seen in Figure 4(a), although ILH do not have specific absorption peak within NIR light, it has a strong absorption during the whole NIR region, which provides the possibility of producing PA signal. In vitro PA imaging used to investigate the potential of ILH as a PA contrast agent was implemented using a NIR laser pulses at a wavelength of $808 \mathrm{~nm}$ which has a small background interference. It was found that ILH could generate strong photoacoustic signal upon exposure to NIR laser pulses and the PA signal increased with increasing concentration of ILH (Figure 4(b)), suggesting the high PA contrast potential of the NPs. We assume that the reason for the strong PA signal intensity of this material, despite the fact that it has no specific absorbance in the NIR region, is benefited from its unique structure. The hollow nanostructure has long been used as a contrasting agent in the acoustic imaging for the acoustic signal can be promoted and enhanced, therefore contrasting to the background $[37,38]$. The big cavity as well as the further introduced liposome can be used to amplify the acoustic signal generated by the PA process; therefore a more desired imaging could be achieved. Although the PA intensity of reconstructed image was decreased along with the decline of concentration, the images of NPs with the concentration of $0.25 \mathrm{mg} / \mathrm{mL}$ remained clearly visible. It provided strong evidence that ILH could act as a potential contrast agent for enhancing the noninvasive PA imaging.

3.6. Biocompatibility of ILH and an Enhanced Cytotoxicity of DOX-Loaded ILH. To test the anticancer efficiency of our formulation, firstly we should examine its biocompatibility. We incubated human hepatoma cells with the nanoparticles at different concentrations for $24 \mathrm{~h}$. The relative cell viabilities were then revealed by MTT assay. As can be seen in Figure 5(a), no significant cytotoxicity was observed. The viability of the cells was only reduced by $16.85 \%$ after exposure to ILH dispersion containing $100 \mu \mathrm{g} / \mathrm{mL}$ particles, indicating that ILH particles were highly biocompatible.

Antitumor bioactivity of various DOX formulations was evaluated using human hepatoma QSG-7703 cells. QSG-7703 cells grown in 96-well plates were exposed to serial dilutions of DOX-loaded ILH (DOX-ILH) and free DOX, and cell viability was determined by the MTT assay as described in materials and methods. The results suggested that DOX loaded ILH presents a stronger cytotoxicity than free DOX (Figure 5(b)). At the concentration of $5 \mu \mathrm{g} / \mathrm{mL}$, the cell viability of free DOX and DOX-loaded ILH is $48.4 \pm 7.7 \%$ and $26.2 \pm 8.4 \%$, respectively; meanwhile, when the concentration changed into $10 \mu \mathrm{g} / \mathrm{mL}$, same tendency was also observed 

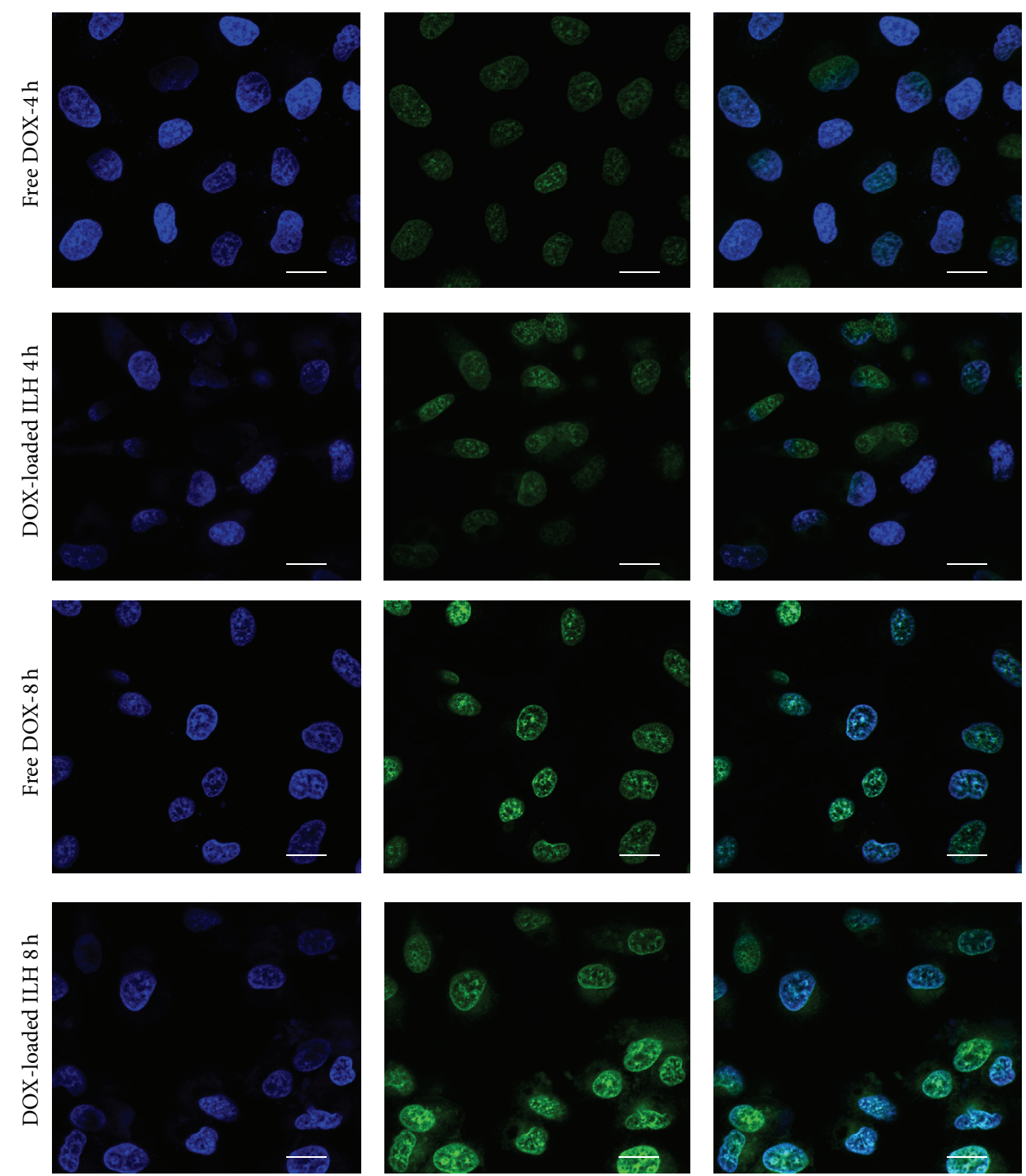

(a)
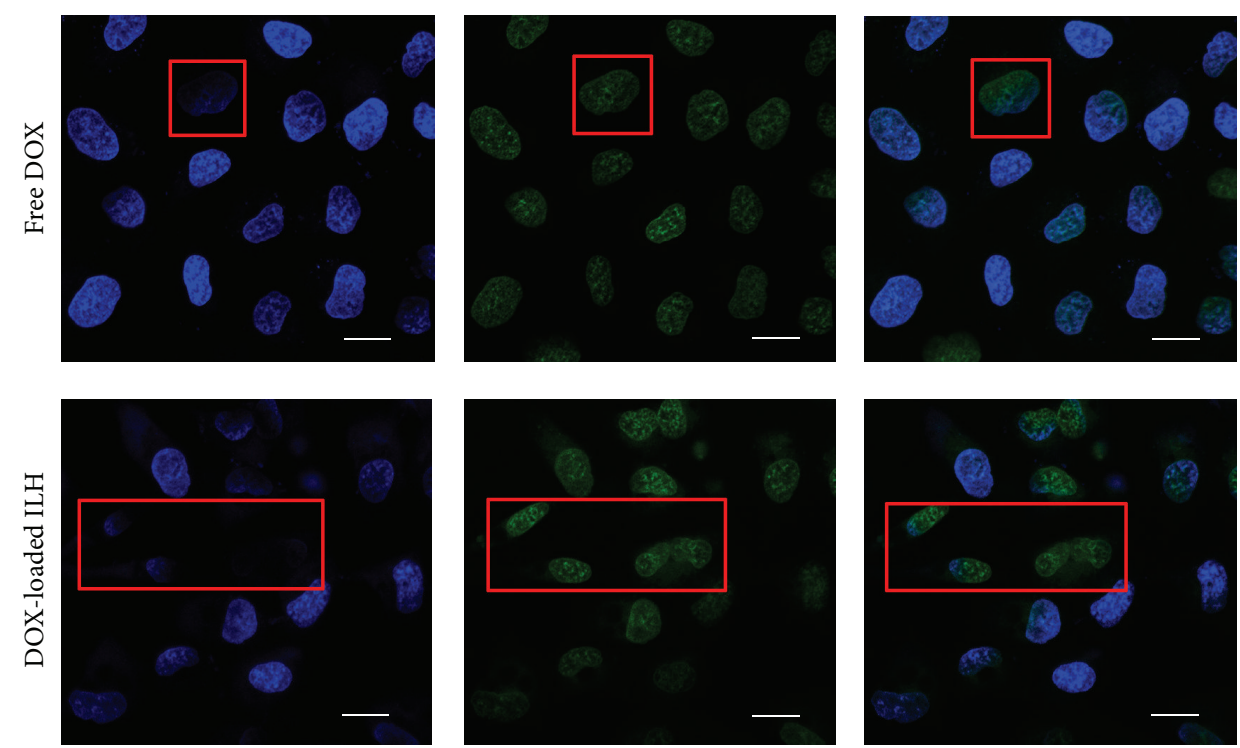

(b)

Figure 6: Continued 

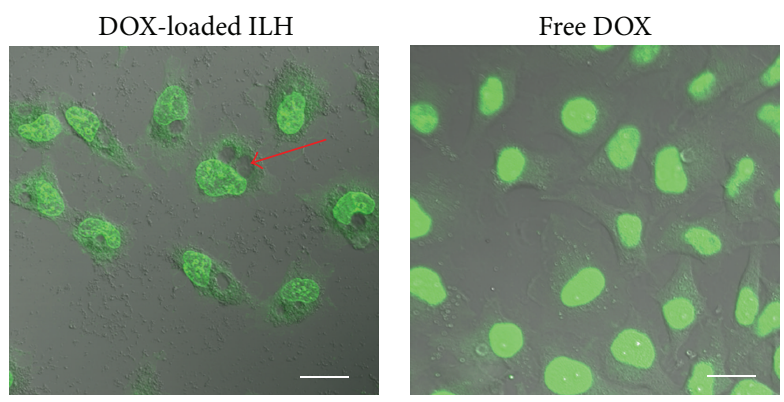

(c)

FIGURE 6: (a) The enhanced DOX internalization by ILH: confocal laser scanning microscopic images of QSG-7703 cells incubated with DOXloaded ILH and free DOX for $4 \mathrm{~h}$ and $8 \mathrm{~h}$. (b) The decreased DAPI staining efficacy indicates the enhanced DOX intracellular trafficking and nucleus localization. (c) QSG-7703 cells (optical microscopy merged with confocal imaging) showed vacuolar degeneration when treated with DOX-ILH after $4 \mathrm{~h}$, indicating an accelerated apoptosis. All the scale bars are $20 \mu \mathrm{m}$.

$(26.2 \pm 6.1 \%, 20.0 \pm 4.5 \%)$. The result can be explained as the DOX-loaded ILH can enter cancer cells more easily than free DOX through endocytosis mechanism [39]. First of all, the DOX loaded nanoparticle can still penetrate the membrane while the free DOX presents lower membrane permeability in tumor environment caused by ion trapping; furthermore, this $\mathrm{pH}$ dependent drug release manner can destroy the endosomal/lysosomal system, and more drug can be leaked to the cytoplasm instead of being degraded. Meanwhile, the function of DOPE [23], which was applied in the liposome, may also contribute to the difference. For years, DOPE has been widely studied because of its excellent fusogenic potential. Several experiments demonstrated that DOPE can enhance the transfection efficiency of cationic liposome due to its capacity to destabilize and/or merge with the cell membrane at acid $\mathrm{pH}$ [24]. Also, its deficiency of a lack of serum stability and nonlamellar property which greatly limits their potential application could be solved by the robust connection with the inner core.

3.7. Cellular Uptake and Intracellular Localization. In order to confirm the antitumor activity of the DOX-loaded ILH, confocal laser scanning microscopic images of cells treated with free DOX and DOX-loaded ILH (at the same DOX concentration of $5 \mu \mathrm{g} / \mathrm{mL}$ ) were observed after $4 \mathrm{~h}$ and $8 \mathrm{~h}$ (Figure 6(a)). The extent of cell uptake of the drug delivery systems plays an important role in the achievement of the required therapeutic efficacy [40]. As doxorubicin is a fluorescent molecule, its internalization by QSG-7703 cells can be followed using fluorescence microscopy. A strong green fluorescence signal was visualized when cells were cultured in the presence of free DOX and also when ILH were used. And the results are consistent with our former assumption. The results clearly show that when treated with ILH, doxorubicin can spread in the cytoplasm of cells after $4 \mathrm{~h}$ of incubation, while the free DOX group exhibits a different phenomenon. It can be explained by a well-known fact that free DOX permeates cellular and nuclear membranes by passive diffusion and tend to rapidly accumulate in the cell nuclei $[41,42]$. From the merged picture we can see that the intensity of green fluorescence in the cell when treated with free DOX was clearly less than DOX-loaded ILH, suggesting that the acidic environment can reduce the free DOX internalization as a result of ion trapping. Conversely, DOX loaded nanoparticles are expected to be taken up by cancer cells via endocytosis. Interestingly, as it is shown in Figure 6(b), we found that comparing to free drug group, DOX-loaded ILH group has a much less DAPI staining efficacy. This phenomenon further explains the enhanced cytotoxicity for ILH formulation. As the mechanism for DOX is by its intercalation with DNA, so it can broke the DNA chain, preventing the DNA double helix from being resealed and thereby stopping the process of replication [43]. Once the DOX has intercalated with DNA, it blocks DAPI bonding with the DNA effectively; therefore the DAPI-fluorescence will not be detected. Furthermore, when the incubation time increased to $8 \mathrm{~h}$, an obvious enhanced amount of DOX can be observed in the nucleus. Compared to free DOX experiments, a higher density of green dots can be seen inside the cells at all the time points. These observations are in agreement with the quantitative data obtained in the previous cell viability assays and can be attributed to a high rate of DOX internalization promoted by the nanohybrid as well as to the facilitated release of DOX from the endosomal/lysosomal compartments.

It also should be noted that comparing to free drug, cells treated with DOX-ILH showed vacuolation, which is a sign of apoptosis (Figure 6(c)). Former studies indicate that some nanoscale drug delivery system can enhance the sensibility of the cancer cells to chemotherapeutic drugs; therefore a more desired treating index could be achieved [44]. So the enhanced cytotoxicity could also be attributed to the function of our formulation. Overall, the fluorescence microscopy analysis also confirms that the antitumor efficacy of DOXloaded ILH is higher than that of an equivalent concentration of free DOX.

\section{Conclusion}

In summary, we developed an easy approach to construct a multifunctional iron silicate@liposome nanohybrid for $\mathrm{pH}$ sensitive doxorubicin delivery and photoacoustic imaging. 
This "zero premature release" feature endues our formulation a relatively high loading efficacy, encapsulation efficiency as well as diminishing the side effects. Furthermore, the formulation exhibited a significantly higher cytotoxicity than free DOX as they can effectively transport doxorubicin across the cell membrane and avoid the drug from being trapped into the endosomal/lysosomal system by "proton sponge" effect, so the obstacle of ion-trapping of DOX could also be solved. The strategy of using coordination bond to fulfill the $\mathrm{pH}$-sensitive controlled drug releasing for killing cancer cells was confirmed with a better efficiency. Meanwhile, the strong PA signal in vitro ensures it as a promising candidate for further application in PAI, which may bring new possibility for its further application in clinical area.

\section{Conflict of Interests}

The authors declare that there is no conflict of interests regarding the publication of this paper.

\section{Acknowledgments}

The research was supported by the Science and Technology Project of Fujian Province, China (no. 2011Y0051), and the Fundamental Research Funds for the Central Universities (no. 2011121057). The authors thank Nanfeng Zheng (State Key Laboratory for Physical Chemistry of Solid Surfaces and Department of Chemistry, College of Chemistry and Chemical Engineering, Xiamen University) for helpful direction on designing and synthesizing the nanoparticle.

\section{References}

[1] R. Minow, R. Benjamin, and J. A. Gottlieb, "Cardiomyopathan overview with determination of risk factors," Cancer Chemotherapy Reports, vol. 6, p. 195, 1975.

[2] J.-M. Escoffre, C. Mannaris, B. Geers et al., "Doxorubicin liposome-loaded microbubbles for contrast imaging and ultrasound-triggered drug delivery," IEEE Transactions on Ultrasonics, Ferroelectrics, and Frequency Control, vol. 60, no. 1, pp. 7887, 2013.

[3] Y. Qin, H. Chen, Q. Zhang et al., "Liposome formulated with TAT-modified cholesterol for improving brain delivery and therapeutic efficacy on brain glioma in animals," International Journal of Pharmaceutics, vol. 420, no. 2, pp. 304-312, 2011.

[4] Y. Kamimura, K. Furukawa, D. Kittaka, M. Nishio, K. Hamamura, and S. Fukumoto, "Differential enhancing effects of alpha2,8-sialyltransferase on the cell proliferation and mobility," International Journal of Oncology, vol. 26, no. 2, pp. 337-344, 2005.

[5] M. A. Hussein, L. Wood, E. Hsi et al., "A phase II trial of pegylated liposomal doxorubicin, vincristine, and reduced-dose dexamethasone combination therapy in newly diagnosed multiple myeloma patients," Cancer, vol. 95, no. 10, pp. 2160-2168, 2002.

[6] J.-Z. Du, X.-J. Du, C.-Q. Mao, and J. Wang, "Tailor-made dual $\mathrm{pH}$-sensitive polymer-doxorubicin nanoparticles for efficient anticancer drug delivery," Journal of the American Chemical Society, vol. 133, no. 44, pp. 17560-17563, 2011.
[7] Z. Teng, X. Zhu, G. Zheng et al., "Ligand exchange triggered controlled-release targeted drug delivery system based on coreshell superparamagnetic mesoporous microspheres capped with nanoparticles," Journal of Materials Chemistry, vol. 22, no. 34, pp. 17677-17684, 2012.

[8] J. W. Wojtkowiak, D. Verduzco, K. J. Schramm, and R. J. Gillies, "Drug resistance and cellular adaptation to tumor acidic $\mathrm{pH}$ microenvironment," Molecular Pharmaceutics, vol. 8, no. 6, pp. 2032-2038, 2011.

[9] B. P. Mahoney, N. Raghunand, B. Baggett, and R. J. Gillies, "Tumor acidity, ion trapping and chemotherapeutics: I. Acid $\mathrm{pH}$ affects the distribution of chemotherapeutic agents in vitro," Biochemical Pharmacology, vol. 66, no. 7, pp. 1207-1218, 2003.

[10] L. Jing, X. Liang, Z. Deng et al., "Prussian blue coated gold nanoparticles for simultaneous photoacoustic/CT bimodal imaging and photothermal ablation of cancer," Biomaterials, vol. 35, no. 22, pp. 5814-5821, 2014.

[11] J. V. Jokerst, D. van de Sompel, S. E. Bohndiek, and S. S. Gambhir, "Cellulose nanoparticles are a biodegradable photoacoustic contrast agent for use in living mice," Photoacoustics, vol. 2, no. 3, pp. 119-127, 2014.

[12] H. J. Lee, Y. Liu, J. Zhao et al., "In vitro and in vivo mapping of drug release after laser ablation thermal therapy with doxorubicin-loaded hollow gold nanoshells using fluorescence and photoacoustic imaging," Journal of Controlled Release, vol. 172, no. 1, pp. 152-158, 2013.

[13] J. M. Tam, J. O. Tam, A. Murthy et al., "Controlled assembly of biodegradable plasmonic nanoclusters for near-infrared imaging and therapeutic applications," ACS Nano, vol. 4, no. 4, pp. 2178-2184, 2010

[14] J. F. Lovell, C. S. Jin, E. Huynh et al., "Porphysome nanovesicles generated by porphyrin bilayers for use as multimodal biophotonic contrast agents," Nature Materials, vol. 10, no. 4, pp. 324332,2011

[15] W. Fang, J. Yang, J. Gong, and N. Zheng, "Photo- and pHtriggered release of anticancer drugs from mesoporous silicacoated Pd@Ag nanoparticles," Advanced Functional Materials, vol. 22, no. 4, pp. 842-848, 2012.

[16] C. Barbé, J. Bartlett, L. Kong et al., "Silica particles: a novel drugdelivery system," Advanced Materials, vol. 16, no. 21, pp. 19591966, 2004.

[17] D. R. Radu, C.-Y. Lai, K. Jeftinija, E. W. Rowe, S. Jeftinija, and V. S.-Y. Lin, "A polyamidoamine dendrimer-capped mesoporous silica nanosphere-based gene transfection reagent," Journal of the American Chemical Society, vol. 126, no. 41, pp. 13216-13217, 2004.

[18] Z. Luo, Y. Hu, K. Cai et al., "Intracellular redox-activated anticancer drug delivery by functionalized hollow mesoporous silica nanoreservoirs with tumor specificity," Biomaterials, vol. 35, no. 27, pp. 7951-7962, 2014.

[19] S. Santra, P. Zhang, K. Wang, R. Tapec, and W. Tan, “Conjugation of biomolecules with luminophore-doped silica nanoparticles for photostable biomarkers," Analytical Chemistry, vol. 73, no. 20, pp. 4988-4993, 2001.

[20] A. Kheirolomoom, L. M. Mahakian, C.-Y. Lai et al., "Copperdoxorubicin as a nanoparticle cargo retains efficacy with minimal toxicity," Molecular Pharmaceutics, vol. 7, no. 6, pp. 19481958, 2010.

[21] S. Majumder, P. Dutta, A. Mookerjee, and S. K. Choudhuri, "The role of a novel copper complex in overcoming doxorubicin resistance in Ehrlich ascites carcinoma cells in vivo," ChemicoBiological Interactions, vol. 159, no. 2, pp. 90-103, 2006. 
[22] X. Xu, H. L. Persson, and D. R. Richardson, "Molecular pharmacology of the interaction of anthracyclines with iron," Molecular Pharmacology, vol. 68, no. 2, pp. 261-271, 2005.

[23] H. Farhood, N. Serbina, and L. Huang, "The role of dioleoyl phosphatidylethanolamine in cationic liposome mediated gene transfer," Biochimica et Biophysica Acta, vol. 1235, no. 2, pp. 289295, 1995.

[24] J. Shin, P. Shum, and D. H. Thompson, "Acid-triggered release via dePEGylation of DOPE liposomes containing acid-labile vinyl ether PEG-lipids," Journal of Controlled Release, vol. 91, no. 1-2, pp. 187-200, 2003.

[25] P. Liu, M. Chen, C. Chen, X. Fang, X. Chen, and N. Zheng, "An iron silicate based $\mathrm{pH}$-sensitive drug delivery system utilizing coordination bonding," Journal of Materials Chemistry B, vol. 1, no. 22, pp. 2837-2842, 2013.

[26] M. Rozenberg, A. Loewenschuss, and Y. Marcus, "IR spectra and hydration of short-chain polyethyleneglycols," Spectrochimica Acta-Part A: Molecular and Biomolecular Spectroscopy, vol. 54, no. 12, pp. 1819-1826, 1998.

[27] M. Mrlík, M. Ilčíková, V. Pavlínek, J. Mosnáček, P. Peer, and P. Filip, "Improved thermooxidation and sedimentation stability of covalently-coated carbonyl iron particles with cholesteryl groups and their influence on magnetorheology," Journal of Colloid and Interface Science, vol. 396, pp. 146-151, 2013.

[28] Y. Qian, P. Wei, P. Jiang, Z. Li, Y. Yan, and J. Liu, "Preparation of a novel PEG composite with halogen-free flame retardant supporting matrix for thermal energy storage application," Applied Energy, vol. 106, pp. 321-327, 2013.

[29] J. Hrkach, D. von Hoff, M. M. Ali et al., "Preclinical development and clinical translation of a PSMA-targeted docetaxel nanoparticle with a differentiated pharmacological profile," Science Translational Medicine, vol. 4, no. 128, Article ID 128ra39, 2012.

[30] T. S. Levchenko, R. Rammohan, A. N. Lukyanov, K. R. Whiteman, and V. P. Torchilin, "Liposome clearance in mice: the effect of a separate and combined presence of surface charge and polymer coating," International Journal of Pharmaceutics, vol. 240, no. 1-2, pp. 95-102, 2002.

[31] K. Xiao, Y. Li, J. Luo et al., "The effect of surface charge on in vivo biodistribution of PEG-oligocholic acid based micellar nanoparticles," Biomaterials, vol. 32, no. 13, pp. 3435-3446, 2011.

[32] A. Akinc, M. Thomas, A. M. Klibanov, and R. Langer, "Exploring polyethylenimine-mediated DNA transfection and the proton sponge hypothesis," The Journal of Gene Medicine, vol. 7, no. 5, pp. 657-663, 2005.

[33] U. Lächelt, P. Kos, F. M. Mickler et al., "Fine-tuning of proton sponges by precise diaminoethanes and histidines in pDNA polyplexes," Nanomedicine: Nanotechnology, Biology, and Medicine, vol. 10, no. 1, pp. 35-44, 2014.

[34] D. W. Pack, A. S. Hoffman, S. Pun, and P. S. Stayton, "Design and development of polymers for gene delivery," Nature Reviews Drug Discovery, vol. 4, no. 7, pp. 581-593, 2005.

[35] P. K. Jain, K. S. Lee, I. H. El-Sayed, and M. A. El-Sayed, "Calculated absorption and scattering properties of gold nanoparticles of different size, shape, and composition: applications in biological imaging and biomedicine," Journal of Physical Chemistry B, vol. 110, no. 14, pp. 7238-7248, 2006.

[36] G. P. Luke, D. Yeager, and S. Y. Emelianov, "Biomedical applications of photoacoustic imaging with exogenous contrast agents," Annals of Biomedical Engineering, vol. 40, no. 2, pp. 422-437, 2012.
[37] L. An, H. Hu, J. Du et al., "Paramagnetic hollow silica nanospheres for in vivo targeted ultrasound and magnetic resonance imaging," Biomaterials, vol. 35, no. 20, pp. 5381-5392, 2014.

[38] E. G. Schutt, D. H. Klein, R. M. Mattrey, and J. G. Riess, "Injectable microbubbles as contrast agents for diagnostic ultrasound imaging: the key role of perfluorochemicals," Angewandte Chemie - International Edition, vol. 42, no. 28, pp. 32183235, 2003.

[39] W. Luo, W. Ye, and Z. Haiying, "Advances in the researches on endocytosis and cellular localization of nanomaterials and associated mechanisms," Chinese Journal of Cell Biology, vol. 35, pp. 1826-1831, 2013.

[40] J. L. Santos, H. Oliveira, D. Pandita et al., "Functionalization of poly(amidoamine) dendrimers with hydrophobic chains for improved gene delivery in mesenchymal stem cells," Journal of Controlled Release, vol. 144, no. 1, pp. 55-64, 2010.

[41] M. Shi, K. Ho, A. Keating, and M. S. Shoichet, "Doxorubicinconjugated immuno-nanoparticles for intracellular anticancer drug delivery," Advanced Functional Materials, vol. 19, no. 11, pp. 1689-1696, 2009.

[42] J. A. MacKay, M. Chen, J. R. McDaniel, W. Liu, A. J. Simnick, and A. Chilkoti, "Self-assembling chimeric polypeptide-doxorubicin conjugate nanoparticles that abolish tumours after a single injection," Nature Materials, vol. 8, no. 12, pp. 993-999, 2009.

[43] O. Tacar, P. Sriamornsak, and C. R. Dass, "Doxorubicin: an update on anticancer molecular action, toxicity and novel drug delivery systems," Journal of Pharmacy and Pharmacology, vol. 65, no. 2, pp. 157-170, 2013.

[44] Q. Zhang, W. Yang, N. Man et al., "Autophagy-mediated chemosensitization in cancer cells by fullerene C60 nanocrystal," Autophagy, vol. 5, no. 8, pp. 1107-1117, 2009. 

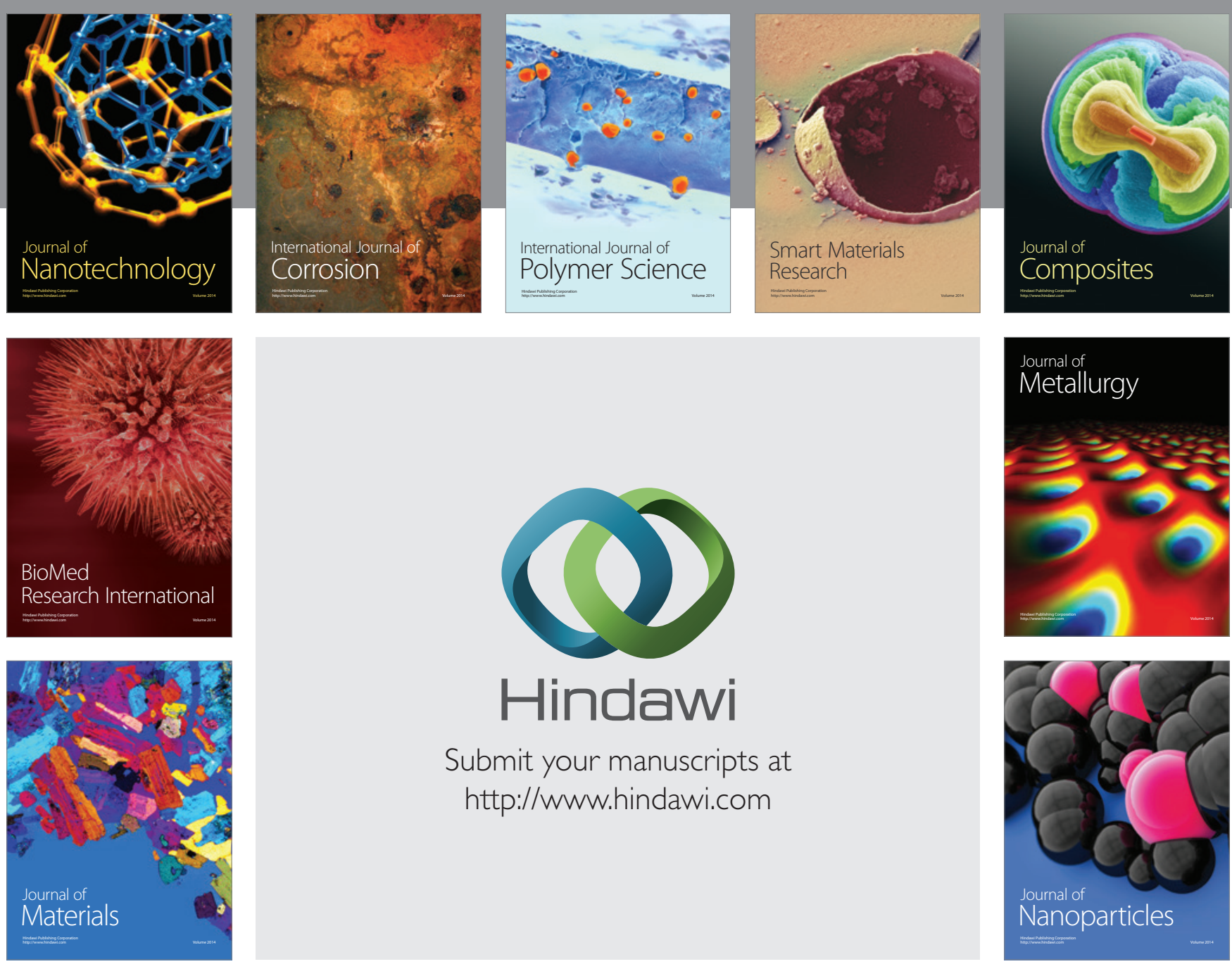

Submit your manuscripts at http://www.hindawi.com
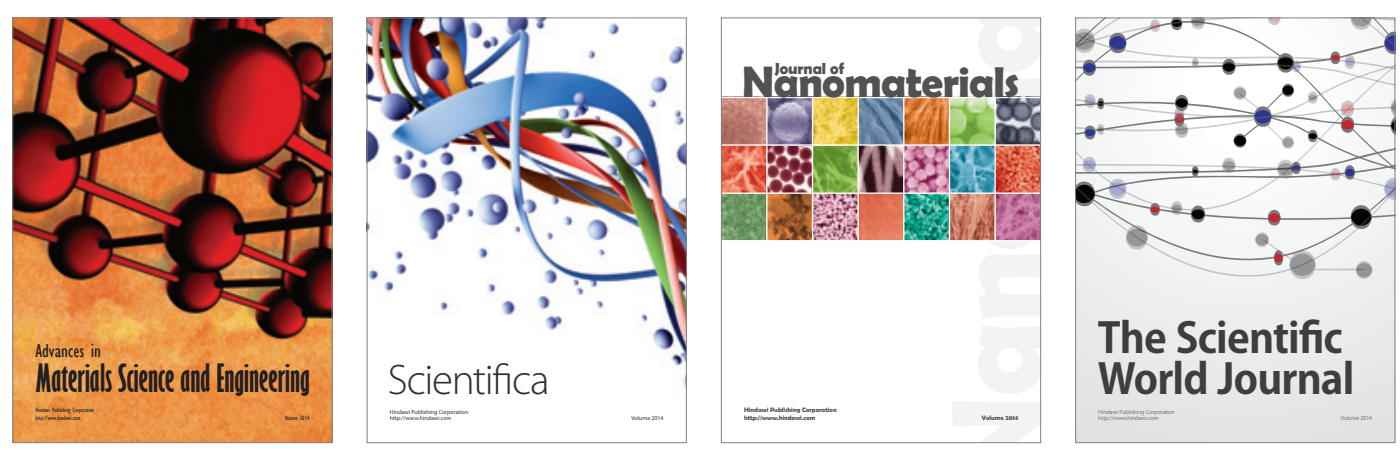

\section{The Scientific World Journal}
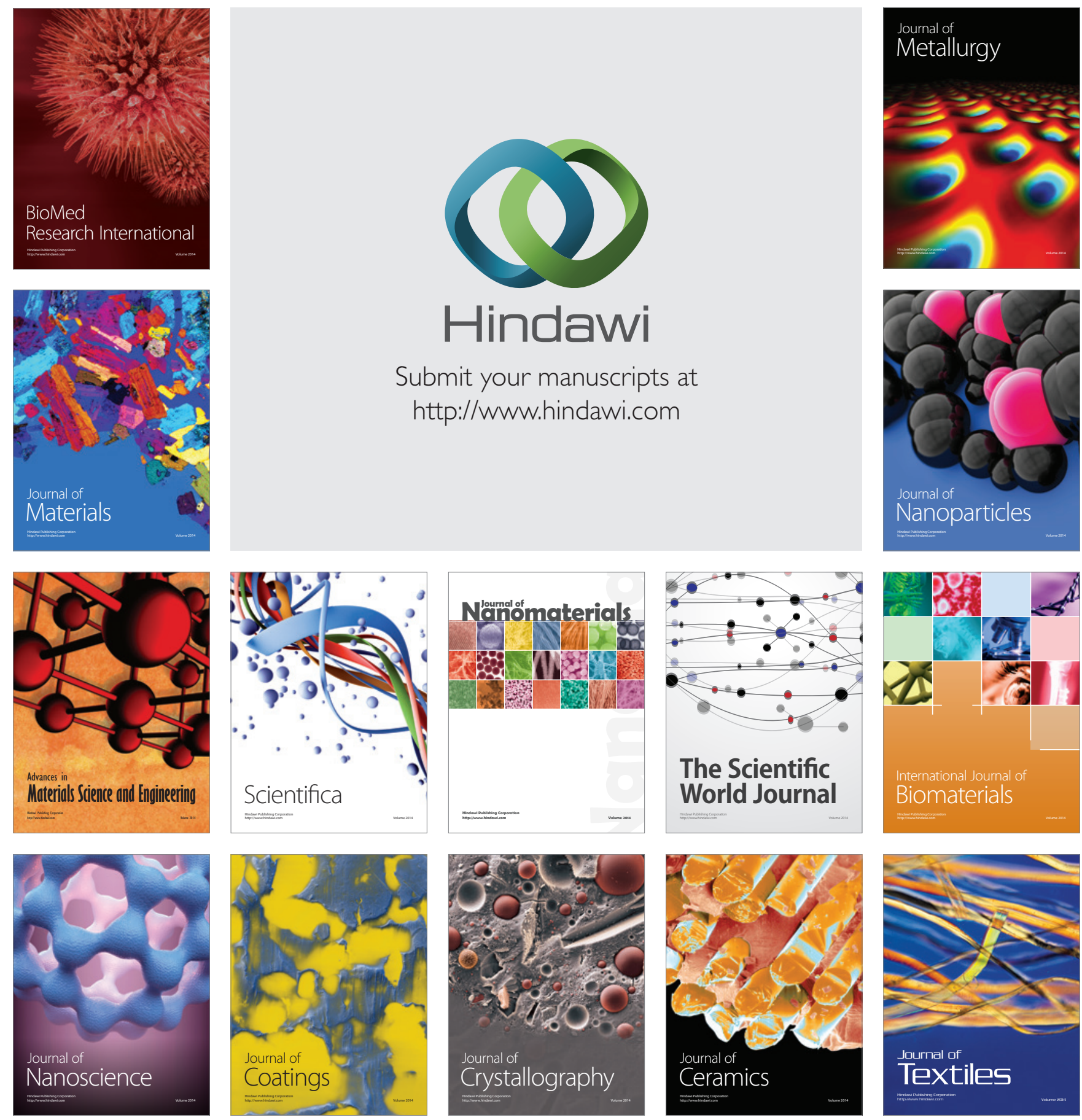